\title{
Qualidade de frutos de tangerina 'Ponkan' utilizando recobrimento alternativos e aplicação de radiação UV-C
}

\author{
Quality of 'Ponkan' mandarin fruits using alternative coatings and application of UV-C radiation \\ Calidad de los frutos de mandarina 'Ponkan' mediante recubrimientos alternativos y aplicación de \\ radiación UV-C
}

Recebido: 02/01/2022 | Revisado: 07/01/2022 |Aceito: 15/01/2022 | Publicado: 17/01/2022

Camila Schwartz Dias

ORCID: https://orcid.org/0000-0002-0061-7874 Universidade Federal de Pelotas, Brasil E-mail: camilaschdias@hotmail.com

Mariana Larrondo Bicca

ORCID: https://orcid.org/0000-0003-0486-4255 Universidade Federal de Pelotas, Brasil

E-mail: mary.bicca@hotmail.com

Flávia Lourenço da Silva

ORCID: https://orcid.org/0000-0002-1890-6420 Universidade Federal de Pelotas, Brasil

E-mail: flavia.lourencodasilva@hotmail.com

Tâmara Foster Acosta

ORCID: https://orcid.org/0000-0002-8116-0393 Universidade Federal de Pelotas, Brasil E-mail: tamaraacosta1986@gmail.com

Gabrielle Leivas

ORCID: https://orcid.org/0000-0003-2671-2316 Universidade Federal de Pelotas, Brasil

E-mail: gabrielleleivas@gmail.com

Paulo Celso de Mello Farias

ORCID: https://orcid.org/0000-0002-6519-4972 Universidade Federal de Pelotas, Brasil

E-mail: mellofarias@yahoo.com.br

Vagner Brasil Costa

ORCID: https://orcid.org/0000-0002-0406-2044 Universidade Federal de Pelotas, Brasil E-mail: vagnerbrasil@gmail.com

Flávio Gilberto Herter

ORCID: https://orcid.org/0000-0001-9652-1756 Universidade Federal de Pelotas, Brasil E-mail: flavioherter@gmail.com

\begin{abstract}
Resumo
As tangerinas representam o segundo maior grupo de frutos cítricos produzidos mundialmente. No Brasil, sua produção está concentrada na região Sudeste e Sul do país. A variedade mais cultivada nos pomares brasileiros é a tangerina 'Ponkan', muito apreciada para o consumo in natura. Nos últimos anos houveram mudanças no perfil dos consumidores e um aumento na busca por alimentos livres de resíduos. Novos métodos de conservação alternativos, como a utilização de recobrimentos e tratamentos físicos no armazenamento dos frutos vem sendo estudados. O objetivo deste trabalho foi avaliar a utilização dos recobrimentos fécula de mandioca e gelatina, aliados a aplicação de radiação UV-C, para prolongar a vida útil de tangerinas 'Ponkan' durante o armazenamento. O experimento foi inteiramente casualizado contendo como fatores de tratamento os recobrimentos fécula de mandioca $3 \%$ e gelatina, a aplicação de UV-C (com e sem) e dias de armazenamento (5, 10 e 15). Os resultados demonstraram que houve aumento do $\mathrm{pH}$ dos frutos tratados com fécula de mandioca e gelatina, com aplicação de UV-C. A perda de massa dos frutos aumentou com o período de armazenamento e para os SST não houveram diferenças entre os tratamentos. Os atributos de coloração indicam que a fécula de mandioca foi eficiente para manter a coloração dos frutos no armazenamento. Concluiu-se que a utilização de recobrimentos de fécula de mandioca $3 \%$ e gelatina não foram eficientes para manutenção dos atributos químicos de qualidade dos frutos de tangerina 'Ponkan' e a aplicação de radiação UV-C não teve efeito sobre os parâmetros analisados.
\end{abstract}

Palavras-chave: Armazenamento; Citros; Conservação de alimentos; Fécula de mandioca; Gelatina. 


\begin{abstract}
Tangerines represent the second largest group of citrus fruits produced worldwide. In Brazil, its production is concentrated in the Southeast and South regions of the country. The most cultivated variety in Brazilian orchards is the 'Ponkan' mandarin, which is highly appreciated for fresh consumption. In recent years there have been changes in the profile of consumers and an increase in the search for residue-free food. New alternative conservation methods, such as the use of coatings and physical treatments in fruit storage, have been studied. The objective of this work was to evaluate the use of cassava starch and gelatin coatings, combined with the application of UV-C radiation, to prolong the shelf life of 'Ponkan' tangerines during storage. The experiment was completely randomized, containing as treatment factors: $3 \%$ cassava starch and gelatin coatings, UV-C application (with and without) and storage days (5, 10 and 15). The results showed that there was an increase in the $\mathrm{pH}$ of fruits treated with cassava starch and gelatin, with application of UV-C. The loss of fruit mass increased with the storage period and for SST there were no differences between treatments. The color attributes indicate that the cassava starch was efficient in maintaining the fruit color during storage. It was concluded that the use of $3 \%$ cassava starch and gelatin coatings were not efficient in maintaining the chemical quality attributes of 'Ponkan' mandarin fruits and the application of UV-C radiation had no effect on the parameters analyzed.
\end{abstract}

Keywords: Storage; Citrus; Food preservation; Cassava starch; Gelatin.

\title{
Resumen
}

Las mandarinas representan el segundo grupo más grande de frutas cítricas producidas en todo el mundo. En Brasil, su producción se concentra en las regiones Sudeste y Sur del país. La variedad más cultivada en los huertos brasileños es la mandarina 'Ponkan', muy apreciada para el consumo en fresco. En los últimos años se han producido cambios en el perfil de los consumidores y un aumento en la búsqueda de alimentos libres de residuos. Se han estudiado nuevos métodos alternativos de conservación, como el uso de revestimientos y tratamientos físicos en el almacenamiento de frutos. El objetivo de este trabajo fue evaluar el uso de recubrimientos de almidón y gelatina de mandioca, combinados con la aplicación de radiación UV-C, para prolongar la vida útil de las mandarinas 'Ponkan' durante el almacenamiento. El experimento fue completamente al azar, conteniendo 3\% de almidón de mandioca y recubrimientos de gelatina, aplicación de UV-C (con y sin) y días de almacenamiento (5, 10 y 15) como factores de tratamiento. Los resultados mostraron que hubo un aumento en el $\mathrm{pH}$ de frutos tratados con almidón de mandioca y gelatina, con aplicación de UV-C. La pérdida de masa de frutos aumentó con el período de almacenamiento y para SST no hubo diferencias entre tratamientos. Los atributos de color indican que el almidón de mandioca fue eficiente para mantener el color de la fruta durante el almacenamiento. Se concluyó que el uso de almidón de mandioca al $3 \%$ y recubrimientos de gelatina no fue eficiente para mantener los atributos de calidad química de las frutas de mandarina 'Ponkan' y la aplicación de radiación UV-C no tuvo efecto sobre los parámetros analizados.

Palabras clave: Almacenamiento; Cítricos; Conservación de alimentos; Almidón de mandioca; Gelatina.

\section{Introdução}

As tangerinas são frutas de grande importância econômica e constituem o segundo maior grupo de frutos cítricos e em 2019, foi a oitava fruta mais produzida mundialmente. Atualmente o Brasil está na sétima posição dentre os maiores produtores de tangerinas, com aproximadamente 984,9 mil toneladas cultivados em 52, 8 mil hectares (Andrade, 2021). Sua produção está concentrada na região Sudeste e Sul do país, sendo os estados de São Paulo (337.4 mil ton), Minas Gerais (244.5 mil ton), Paraná (157.6 mil ton) e Rio Grande do Sul (122.2 mil ton) os maiores produtores da fruta, respectivamente (IBGE, 2020). A tangerina da variedade "Ponkan" é originária da Ásia e representa 60\% dos plantios dos pomares brasileiros sendo considerada a "rainha das tangerinas do Brasil", por produzir frutos muito apreciados para o consumo in natura pela facilidade de descascar, assim como, por suas características de coloração, sabor e tamanho (Cruz \& Moreira, 2012, 1991; Andrade Pacheco et al. 2013, Bastos et al. 2014).

Os aspectos de qualidade de frutos e hortaliças representados pela cor, aroma, sabor e textura, conferem ao produto suas características e por isto, necessitam ser analisadas em conjunto. A preferência dos consumidores se deve em função dos aspectos de qualidade dos frutos e para satisfazer as exigências do mercado consumidor, a seleção de técnicas adequadas para a pós-colheita, auxilia a cadeia de comercialização (Bezerra, 2003). Nos últimos anos, as exigências dos consumidores por alimentos livres de resíduos químicos, poluentes e não renováveis, tem trazido novos desafios na procura por estratégias alternativas para manutenção da qualidade de frutos. Neste sentido, a utilização de compostos naturais e tratamentos físicos tem demonstrado resultados satisfatórios em laranjas (Pereira et al., 2014), tangerinas (Barbará et al., 2020) e mangas 
(Scanavaca et al., 2007). A irradiação de frutas e hortaliças por raios gama e UV-C (254nm), tem sido utilizada para controle de fungos que causam podridões na pós-colheita e também para a inativação de patógenos em frutos frescos e legumes minimamente processados, além de serem efetivos na extensão da vida útil dos produtos, retardando os processos fisiológicos e bioquímicos que levam a maturação (Cia et al., 2007; Aranha et al., 2017; Vasconcelos et al. 2020). No entanto, podem ser verificados alguns efeitos adversos como o escurecimento e danos nos tecidos, havendo a necessidade de uma cuidadosa seleção da dose a ser aplicada para cada produto vegetal em particular (Civello et al., 2006).

A utilização de barreiras físicas visa prolongar o tempo de armazenamento dos frutos, preservando suas características que conferem qualidade ao produto. Estas barreiras evitam a perda de água, restringem a difusão dos gases, como o oxigênio e o dióxido de carbono, reduzindo a taxa respiratória, além de evitar a perca dos aromas, ainda, podem introduzir aditivos como antioxidantes e antimicrobianos, que melhoram as características intrínsecas e a integridade mecânica dos vegetais cobertos (Botrel, et al. 2010; Vasconcelos et al. 2020). A cera (lipídeos) é uma das principais matérias primas utilizadas nos recobrimentos de frutas (Flores Cantillano et al. 2011), contudo, outras opções como os polissacarídeos (amido, celulose e seus derivados) têm sido explorados para tal fim (Assis \& Britto, 2014). Os filmes à base de amido apresentam boas propriedades mecânicas e excelente propriedade de barreira ao O2. A fécula de mandioca tem sido amplamente utilizada como matériaprima para coberturas e revestimentos comestíveis (Leme et al. 2007), por apresentar vantagens como, formação de películas transparentes e resistentes, sem aspecto pegajoso (Mohr et al. 2015) além de se tratar de um produto biodegradável e que não causa impacto ambiental, encontrada abundantemente e de baixo custo (Ribeiro et al., 2009; Castañeda, 2013).

Diante do exposto, o objetivo deste trabalho foi avaliar a utilização dos recobrimentos fécula de mandioca e gelatina, aliados a aplicação de radiação UV-C, para prolongar a vida útil de tangerinas 'Ponkan' durante o armazenamento.

\section{Metodologia}

A natureza da pesquisa quanto aos procedimentos técnicos, é classificada como Pesquisa Experimental e foi realizada no mês de junho de 2019 no pomar experimental da Universidade Federal de Pelotas, no município do Capão do Leão-RS. Os frutos da variedade 'Ponkan' foram colhidos de plantas com 12 anos de idade e cultivadas sob espaçamento de 4,00 x 4,50. Na coleta dos frutos, selecionou-se os que apresentavam coloração de casca predominantemente amarela e manteve-se o pedúnculo. Os mesmos foram armazenados em caixa de frutas com dimensões de 30x 36 x 55 e transportados ao laboratório onde se procedeu a desinfestação utilizando uma solução de hipoclorito de sódio na concentração de 5ppm onde os frutos permaneceram durante 10 minutos e posteriormente foram colocados para secar sob temperatura ambiente. Após desinfestação, os frutos foram separados conforme os tratamentos: tratamento UV-C com Fécula de mandioca 3\%, sem UV-C com Fécula de Mandioca 3\%, UV-C com Gelatina, sem UV-C com Gelatina, com UV-C e sem recobrimento, e controle (sem UV-C e sem recobrimento).

Os frutos que pertenciam ao tratamento com UV-C foram expostos a radiação ultravioleta de comprimento de onda de 250nm, durante $10 \mathrm{~min}$ e distantes $20 \mathrm{~cm}$ da fonte emissora de radiação. Logo em seguida, os frutos foram submetidos aos tratamentos com os diferentes recobrimentos. Para o preparo do recobrimento com fécula de mandioca a 3\%, procedeu-se sua dissolução da mesma em água destilada e aquecimento controlado até o ponto de geleificação, aos $70^{\circ} \mathrm{C}$. Posteriormente foi resfriada a temperatura ambiente para aplicação. Para o recobrimento de gelatina, procedeu-se o aquecimento de água destilada até o ponto de fervura, acrescentou-se a gelatina e agitou-se até sua completa dissolução. Para a aplicação dos recobrimentos após o resfriamento das coberturas, os frutos foram completamente imersos nos diferentes recobrimentos, conforme o tratamento e dispostos em uma bancada para secagem sob ventilação forçada e posteriormente foram colocados em caixas de fruta armazenados sob temperatura ambiente para simular a condição de comercialização em gôndolas de supermercado $(25 \pm$ $\left.5^{\circ} \mathrm{C}, \mathrm{UR}: 75 \pm 4 \%\right)$. 
As variáveis determinadas foram:

-Coloração dos frutos: foi determinada através do colorímetro digital da marca Minolta modelo CR 300, sistema de leitura CIE $\mathrm{L}^{*} \mathrm{a}^{*} \mathrm{~b}^{*}$, onde o $\mathrm{L}^{*}$ se refere a luminosidade (preto - branco), o a* indica o teor de verde - vermelho e o $\mathrm{b}^{*}$, o teor de azul - amarelo. $\mathrm{O}$ ângulo Hue $\left(\mathrm{H}^{\circ}\right)$ foi obtido a partir das leituras de $\mathrm{a}^{*}$ e $\mathrm{b}^{*}$, através da fórmula arco tangente $\mathrm{b}^{*} / \mathrm{a}^{*}$ (Minolta, 1994), também foi possível obter o croma, que indica a intensidade da coloração. Foram obtidas duas leituras na região equatorial dos frutos.

•Perda de massa (PM): determinada em balança de precisão com três dígitos decimais após a virgula, obtida pela diferença entre a massa inicial dos frutos e a massa final para cada tratamento aos 5, 10 e 15 dias de armazenamento, expressa em porcentagem.

-Potencial hidrogeniônico $(\mathrm{pH})$ : foi avaliado com o potenciômetro digital a partir do suco extraído dos frutos.

-Sólidos solúveis totais (SST): utilizou-se uma amostra de suco dos frutos, e o resultado foi obtidos por leitura direta utilizando o refratômetro digital da marca Atago, expresso em ${ }^{\circ}$ Brix.

O delineamento experimental foi inteiramente casualizado, contendo quatro repetições por tratamento e cada repetição era composta por cinco frutos, tendo como fatores de tratamento os recobrimentos: fécula de mandioca 3\% e aplicação de UVC, fécula de mandioca sem aplicação de UV-C, Gelatina com aplicação de UV-C, Gelatina sem aplicação de UV-C, controle (sem UV-C e sem recobrimento) e três períodos de armazenamento: 5, 10 e 15 dias. Os dados foram submetidos a análise de variância e comparados pelo teste de Tukey a 5\% de probabilidade e quando houve interação significativa entre os tratamentos, a mesma foi considerada na análise.

\section{Resultados e Discussão}

O resultado para a variável Sólidos solúveis não apresentou diferença significativa para os tratamentos e para os dias de armazenamento (Tabela 1). Em virtude do seu metabolismo não climatérico, frutos cítricos não apresentam variações significativas nos teores de açúcares durante o período de armazenamento (Chitarra \& Chitarra, 2005), logo, o recomendado é que sejam colhidos ao atingir o ponto de maturidade fisiológica. Silva et al., (2012) observaram, para esta mesma variedade, que houve um aumento no teor de sólidos solúveis utilizando o recobrimento à base de fécula de mandioca na concentração 1\% e filme PVC em comparação com o tratamento controle (sem recobrimento). Da mesma forma, Vale et al., (2006), avaliando o comportamento de tangerinas Ponkan sob armazenamento refrigerado, verificaram que o teor de sólidos solúveis apresentou variação entre 10.01 a $14.04{ }^{\circ}$ Brix. 
Tabela 1: Valores de sólidos solúveis totais ( ${ }^{\circ}$ Brix) e Perda de massa (\%) de tangerinas 'Ponkan' em função do recobrimento aplicado, com ou sem aplicação de radiação UV-C ao longo do armazenamento.

\begin{tabular}{lll}
\hline Recobrimento & STT & $\begin{array}{l}\text { Perda de } \\
\text { massa (\%) }\end{array}$ \\
\hline Fécula + UVC & $11.416 \mathrm{a}$ & $10.672 \mathrm{~ns}$ \\
Fécula - UVC & $11.583 \mathrm{a}$ & $10.972 \mathrm{~ns}$ \\
Gelatina + UVC & $10.966 \mathrm{a}$ & $10.278 \mathrm{~ns}$ \\
Gelatina - UVC & $10.95 \mathrm{a}$ & $10.461 \mathrm{~ns}$ \\
Controle & $11.775 \mathrm{a}$ & $11.229 \mathrm{~ns}$ \\
\hline Valor de p & 0.03 & 0.81 \\
\hline & & Perda de \\
Armazenamento (dias) & SST & massa (\%) \\
\hline 5 & $11.325 \mathrm{~ns}$ & $5.541 \mathrm{c}$ \\
10 & $11.405 \mathrm{~ns}$ & $10.265 \mathrm{~b}$ \\
15 & $11.285 \mathrm{~ns}$ & $16.362 \mathrm{a}$ \\
\hline Valor de p & 0.88 & $<0.01$ \\
\hline *Letras minúsculas distintas, indicam diferença & estatística na \\
coluna. ns Não significativo. Laboratório de Qualidade de Frutas - \\
UFPel. Capão do Leão, junho de 2019. Fonte: Autores.
\end{tabular}

Quando analisada a variável perda de massa, não foram encontradas diferenças significativas entre os distintos recobrimentos (Tabela 1), contudo, verificou-se diferenças com relação ao tempo de armazenamento. Aos 15 dias de armazenamento, a perda de massa atingiu $16.36 \%$, diferindo estatisticamente dos dias 5 e 10 , os quais apresentaram perdas de $5.54 \%$ e $10.26 \%$, respectivamente. Avaliando morangos da cultivar San Andreas submetidos a aplicação UV-C e armazenados em diferentes temperaturas, Marques et al., (2020) obtiveram maiores valores para perda de massa em um período de seis dias de armazenamento a uma temperatura de $18{ }^{\circ} \mathrm{C}$. Conforme mencionado no parágrafo anterior, a tangerina, assim como o morango, pertence ao grupo de frutos não climatéricos, e neste caso, a perda de massa se justifica pelo processo de transpiração, o qual resulta de um gradiente de pressão de vapor entre o fruto e o ambiente. Muitas pesquisas relatam o uso de ceras como uma alternativa para interferir neste gradiente, a fim de reduzir a perda de massa fresca e manter a qualidade dos frutos durante mais tempo; contudo, com o avanço do período de armazenamento também são verificadas perdas ocasionadas pela deterioração dos frutos (Vale et al., 2006; Barbará et al., 2020). 
Tabela 2. Valores de pH de tangerinas 'Ponkan' em função do recobrimento aplicado, com ou sem aplicação de radiação UVC, ao longo do armazenamento.

\begin{tabular}{l|lll}
\cline { 3 - 4 } & \multicolumn{3}{c}{ Valores de $\mathrm{pH}$} \\
\cline { 2 - 4 } Recobrimento & 5 & 10 & 15 \\
\hline Fécula+UVC & $4.04 \mathrm{abB}$ & $4.19 \mathrm{aAB}$ & $4.27 \mathrm{aA}$ \\
Fécula-UVC & $4.12 \mathrm{aA}$ & $3.90 \mathrm{bB}$ & $4.20 \mathrm{abA}$ \\
Gelatina+UVC & $3.86 \mathrm{bcB}$ & $4.11 \mathrm{aA}$ & $4.04 \mathrm{bcAB}$ \\
Gelatina-UVC & $3.92 \mathrm{~b}$ & $3.90 \mathrm{~b}$ & $3.90 \mathrm{c}$ \\
Controle & $3.70 \mathrm{cA}$ & $3.88 \mathrm{bA}$ & $3.85 \mathrm{cA}$ \\
\hline
\end{tabular}

*Letras minúsculas distintas, demonstram diferenças estatísticas na coluna. Letras maiúsculas distintas, demonstram diferenças estatísticas na linha. Laboratório de Qualidade de Frutas - UFPel. Capão do Leão, junho de 2019. Fonte: Autores.

Considerando-se a avaliação do $\mathrm{pH}$, houve interação significativa entre os fatores de tratamento recobrimentos comestíveis e dias de armazenamento (Tabela 2). Os valores médios variaram entre 3.7 a 4.27 , e o menor valor de pH obtido aos 15 dias de armazenamento foi observado na utilização do recobrimento de gelatina sem aplicação UV-C, não diferindo estatisticamente do controle. Já os maiores valores foram obtidos nos tratamentos com fécula de mandioca com e sem aplicação UV-C (4.27 e 4.20, respectivamente). Os resultados se assemelham àqueles obtidos por Dotto et al., (2015), que, dentre os tratamentos utilizados, obtiveram o maior valor de $\mathrm{pH}$ com o recobrimento de fécula de mandioca a $3 \%$ em limas Tahiti armazenadas em caixa plástica. Avaliando recobrimentos à base de fécula de mandioca e óleo de copaíba em goiabas da cultivar Paluma e Mello (2016) obteve maiores valores de pH para fécula de mandioca na concentração de 3\%. O pH está associado a determinação de ácidos no alimento, e sua medida refere-se a concentração de prótons de hidrogênio $\left(\mathrm{H}^{+}\right)$no suco dos frutos. Logo, de forma simplificada, o pH expressa o ácido dissociado que tem a capacidade tamponante (Costa, 2009).

Para o tempo de armazenamento, de modo geral, observou-se o aumento nos valores de pH, com exceção da testemunha, que não mostrou variação significativa em seus valores ao longo dos dias de armazenamento. Segundo Chitarra \& Chitarra (2005), considera-se o pH um indicativo do nível de deterioração do fruto: o aumento gradativo nos seus índices é decorrente da redução da acidez devido ao processo de amadurecimento. A aplicação do recobrimento de fécula de mandioca, alterou a atmosfera ao redor do fruto, reduzindo a permeabilidade e dificultando a troca de gases com o ambiente. Nesta situação, os níveis de $\mathrm{CO}_{2}$ se elevaram e de $\mathrm{O}_{2}$ reduziram, resultando em um aumento da taxa de respiração anaeróbica e induzindo o processo fermentativo.

Com relação a coloração da epiderme dos frutos, os valores de a* variaram quanto ao recobrimento utilizado aos 15 dias de armazenamento (Tabela 3). Foi possível verificar que os frutos recobertos com fécula de mandioca com e sem aplicação de UV-C apresentaram coloração mais próxima ao verde quando comparados ao tratamento controle. Para a coordenada $b^{*}$, aos 15 dias de armazenamento, os frutos do tratamento controle apresentaram coloração mais amarela que os frutos recobertos com fécula de mandioca.

Ao analisar a variável ângulo Hue (Tabela 3), aos 15 dias verificou-se diferenças significativas entre os recobrimentos utilizados e os valores dos tratamentos fécula de mandioca com UV-C e fécula de mandioca sem UV-C, sendo que apenas o primeiro diferiu do tratamento controle (Tabela 3). Houve uma redução na manutenção da coloração para os frutos do controle (76.32), ainda que não diferindo estatisticamente dos recobrimentos de Gelatina com UV-C (77.53) e sem UVC (80.13) (Tabela 3). A fécula de mandioca proporcionou a maior eficiência na manutenção da cor durante o período avaliado, entretanto não diferiu estatisticamente dos tratamentos de recobrimento de Gelatina, independentemente da utilização ou não da radiação 
UV-C. Já Marques et al., (2020) observaram em seu trabalho que os morangos tratados com radiação UV-C mantiveram a coloração vermelha com maior eficiência até o final do período avaliado, quando comparados àqueles que não foram irradiados com UV-C. Para Ferreira e Spricigo (2017), a cor é um importante atributo na agricultura, principalmente tratandose de frutas e hortaliças, pois para o consumidor final a coloração está associada à qualidade do produto, determinando, muitas vezes, o seu valor de mercado.

Tabela 3: Valores de $\mathrm{a}^{*}, \mathrm{~b} * \mathrm{e}$ ângulo Hue de tangerinas Ponkan armazenadas com diferentes recobrimentos, com ou sem aplicação de UVC. Laboratório de qualidade de frutas- UFPel. Capão do Leão, junho de 2019.

\begin{tabular}{|c|c|c|c|c|c|c|c|c|c|c|c|c|}
\hline \multirow[b]{3}{*}{ Recobrimento } & \multicolumn{3}{|c|}{$a^{*}$} & \multicolumn{3}{|c|}{$b^{4}$} & \multicolumn{3}{|c|}{ Hue } & \multicolumn{3}{|c|}{ Croma } \\
\hline & \multicolumn{3}{|c|}{ Armazenamento } & \multicolumn{3}{|c|}{ Arnazenamento } & \multicolumn{3}{|c|}{ Arnazenemento } & \multicolumn{3}{|c|}{ Armazenaumento } \\
\hline & 5 & 10 & 15 & 5 & 10 & 15 & 5 & 10 & 15 & 5 & 10 & 15 \\
\hline Fécula-UVC & $10.43 a b$ & 7.49 & $6.90 \mathrm{~b}$ & $60.44 \mathrm{abA}$ & $53.44 \mathrm{~B}$ & $51.0 \$ b B$ & $8021 b$ & 82.02 & $82.61 \mathrm{a}$ & 61.36 abA & $53.97 \mathrm{~B}$ & $51.61 \mathrm{bB}$ \\
\hline Fécula_UVC & $8.47 a b$ & 12.08 & $8.56 \mathrm{~b}$ & $57.49 \mathrm{abA}$ & $56.79 \mathrm{~A}$ & $48.02 \mathrm{bB}$ & $81.68=b$ & 78.22 & $80.29 \mathrm{ab}$ & $58.14 \mathrm{abA}$ & $58.16 \mathrm{~A}$ & $48.59 \mathrm{bB}$ \\
\hline Gelatina+UVC & $8.0 \mathrm{ab}$ & 9.97 & $12.01 \mathrm{sb}$ & $54.71 b$ & 55.65 & $54.13 \mathrm{ab}$ & $81.72 \mathrm{abA}$ & $799 \mathrm{AB}$ & $77.53 \mathrm{bB}$ & $55.32 \mathrm{~b}$ & 56.55 & $55.47 \mathrm{ab}$ \\
\hline Gelatima-UVC & $4.43 \mathrm{bB}$ & $9.31 \mathrm{~A}$ & 9.77abA & $53.31 b$ & 56.59 & $55.34 \mathrm{ab}$ & $85.29 \mathrm{aA}$ & $80.95 B$ & $80.13 \mathrm{sbB}$ & $53.92 \mathrm{~b}$ & 57.47 & $56.26 \mathrm{ab}$ \\
\hline Controle & $12.23 a$ & 12.03 & $15.17 \mathrm{a}$ & $63.71 a$ & 58.22 & 62.130 & $79.17 \mathrm{~b}$ & 78.42 & $76.32 \mathrm{~b}$ & $64.88 \mathrm{a}$ & 59.47 & $63.99 \mathrm{a}$ \\
\hline
\end{tabular}

*Letras minúsculas distintas, demonstram diferenças estatísticas na coluna. Letras maiúsculas distintas, demonstram diferenças estatísticas na

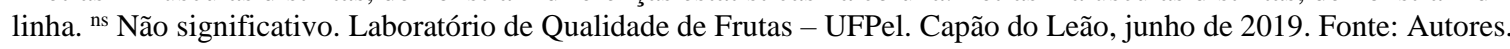

O processo de respiração é afetado pela maior concentração de $\mathrm{CO}_{2}$ presente na atmosfera controlada que os diferentes recobrimentos proporcionam aos frutos durante o período de armazenamento. Durante a respiração ocorre a conversão dos carboidratos, ácidos e lipídeos em $\mathrm{CO}_{2}$ e água (Tavares, 2003). O aumento do $\mathrm{CO}_{2}$ nestas condições possibilita a redução ou até mesmo a inibição da síntese de etileno, fitormônio que promove mudanças na coloração da epiderme dos frutos (Assmann et al., 2006). Os resultados de Damasceno et al., (2003) ao trabalhar com fécula de mandioca a 3\% em tomates, evidenciaram melhor aparência dos frutos tratados em relação à testemunha, no entanto houveram diferenças para perda de massa e textura.

O croma apresentou maiores valores para o tratamento controle e não diferiu do tratamento com gelatina com e sem aplicação de UV-C (Tabela 3). Os menores valores para a variável foram obtidos com o uso de fécula de mandioca com e sem aplicação de UV-C, todavia não diferiram estaticamente do tratamento com gelatina independente da aplicação de radiação. Os resultados sugerem que há uma maior preservação da colocação com a aplicação do recobrimento de fécula de mandioca aos 15 dias em relação aos outros tratamentos, contudo, foi observado sua redução ao longo do período de armazenamento. O trabalho de Vasconcelos et al., (2020), verificou um aumento do croma em tangerinas 'Dekopon' tratados com radiação UV-C e também ao longo do período de armazenamento.

\section{Conclusão}

A utilização dos recobrimentos de fécula de mandioca 3\% e gelatina não foram eficientes para preservar as características químicas dos frutos de tangerina Ponkan ao longo dos 15 dias de armazenamento, contudo se demonstraram eficazes para preservar os atributos visíveis dos frutos. A aplicação de radiação UV-C não teve influência nos parâmetros avaliados. Novas pesquisas deverão ser realizadas para ajustar a espessura destes recobrimentos.

Novos estudos serão necessários para encontrar uma concentração adequada de fécula de mandioca e gelatina que proporcionarão a preservação das características químicas dos frutos de tangerina Ponkan, mantendo os atributos visuais. 


\section{Referências}

Andrade, P. (2021) Boletim Semanal. Departamento de Economia Rural (DERAL)- PR, 2021. https://www.agricultura.pr.gov.br/Pagina/Conjuntura-BoletimSemanal-162021

Andrade Pacheco, C., Schinor, E. H., Cristofani-Yaly, M., Verruma-Bernardi, M. R., Machado, M. A., Bastianel, M., de Azevedo, F. A. (2013). Qualidade pós-colheita de um híbrido do tipo Ponkan de maturação tardia. Citrus Research \& Technology, 34 (2), 39-45.

Aranha, J. B.; Negrini, T. C.; Martin, J. G.; Spoto, M. H. (2017) Efeito da radiação gama nos parâmetros microbiológicos, físico-químicos e compostos fenólicos de farinha de resíduos de frutas durante o armazenamento. Brazilian Journal of Food Technology, 20.

Assmann, A. P., Citadin, I., Kalicz, C. A., Locatelli, M. C., \& Danner, M. A. (2006). Armazenamento de caqui cv. Fuyu e laranja cv. Pera em atmosfera modificada sob diferentes temperaturas. Synergismus scyentifica, 1, 133-143.

Assis, O. B. G., \& Britto, D. D. (2014). Revisão: coberturas comestíveis protetoras em frutas: fundamentos e aplicações. Brazilian Journal of Food Technology, 17, 87-97.

Barbará, M. A., da Veiga, J. C., da Silva, B. M. P., de Toledo Valentini, S. R., Cia, P., \& Bron, I. U. (2020). Refrigeração e cera na qualidade pós-colheita da tangerina 'Fremont' IAC-543. Citrus Research \& Technology, 41, 1-10.

Bastos, D. C., Ferreira, E. A., Passos, O. S., de Sá, J. F., Ataíde, E. M., \& Calgaro, M. (2014). Cultivares copa e porta-enxertos para a citricultura brasileira. Informe Agropecuário (35) 281, 36-45. https://ainfo.cnptia.embrapa.br/digital/bitstream/item/117115/1/Debora-214.pdf

Bezerra, V. S. (2003). Pós-colheita de frutos. Embrapa Documentos, 51, 23p. http://www.infoteca.cnptia.embrapa.br/infoteca/handle/doc/905530

Botrel, D. A., Soares, N. D. F. F., Camilloto, G. P., \& Fernandes, R. V. D. B. (2010). Revestimento ativo de amido na conservação pós-colheita de pera Williams minimamente processada. Ciência Rural, 40, 1814-1820.

Castañeda, L. M. F. (2013). Avaliação da quitosana e da fécula de mandioca, aplicada em pós-colheita no recobrimento de maçãs. Tese de Doutoramento. Porto Alegre, Universidade Federal do Rio Grande do Sul, 130p.

Chitarra, M. I. F. \& Chitarra, A. B. Pós-colheita de frutas e hortaliças: fisiologia e manuseio. (2a ed.), UFLA, 2005. 785p.

Cia, P., Pascholati, S. F., Benato, E. A., Camili, E. C., \& Santos, C. A. (2007). Effects of gamma and UV-C irradiation on the postharvest control of papaya anthracnose. Postharvest Biology and Technology, 43 (3), 366-373.

Civello, P. M., Vicente, A. R., Martínez, G. A. (2006). UV-C technology to control postharverst diseases of fruits and vegetables. Recent advances in alternative postharvest technologies to control fungal diseases in fruit and vegetables, 71-102.

Cruz, M. D. C. M., \& Moreira, R. A. (2012). Production regularity of 'Ponkan' mandarin trees submitted to chemical thinning. Semina: Ciências Agrárias, 33 (6), 2163-2171.

Damasceno, S., Oliveira, P. V. S. D., Moro, E., Macedo Jr, E. K., Lopes, M. C., \& Vicentini, N. M. (2003). Efeito da aplicação de película de fécula de mandioca na conservação pós-colheita de tomate. Food Science and Technology, 23, 377-380.

Dotto, M., Pirola, K., Júnior, A. W., Radaelli, J. C., \& Danner, M. A. (2015). Biofilmes e embalagens na conservação pós-colheita de lima ácida Tahiti. Revista Brasileira de Ciências Agrárias, 10 (3), 365-369.

Ferreira, M. D., \& Spricigo, P. C. (2017). Colorimetria-princípios e aplicações na agricultura. In: FERREIRA, M. D. (Ed.técnico). Instrumentação póscolheita em frutas e hortaliças (p. 209-220). São Carlos: Embrapa Instrumentação.

Flores Cantillano, R. F., Galarça, S. P., Treptow, R. D. O., Castro, L. A. S. (2011). Efeito da atmosfera modificada na qualidade pós-colheita de tangerinas "ponkan" durante o armazenamento refrigerado. Embrapa Clima Temperado-Boletim de Pesquisa e Desenvolvimento (INFOTECA-E).

IBGE (2020). https://sidra.ibge.gov.br/tabela/1613

Leme, A., Groppo, V. D., Romero, A. D. C., Spoto, M. H. F., \& Jacomino, A. P. (2007). Influência do uso de películas comestíveis em laranja pêra minimamente processada. Boletim do Centro de Pesquisa e Processamento de Alimentos, 25(1), $15-24$.

Marques, L. O. D, Benati, J. A., Carvalho, J. D., Mello-Farias, P. Acosta, T. F., Herter, F. G. \& Pasa, M. S. (2020). Sustainable alternatives for post-harvest strawberry conservation. Australian Journal of Crop Science, 14 (10), 1670-1674. 10.21475/ajcs.20.14.10.p2550

Mello, R. D. S. (2016). Recobrimentos biodegradáveis a base de fécula de mandioca e óleo de macaíba na conservação pós-colheita de goiaba 'Paluma'. Trabalho de conclusão de curso. Areia, Universidade Federal da Paraíba. 82p.

Minolta (1994). Precise color communication: color control from feeling to instrumentation. MINOLTA Co. Ltd.

Mohr, L., Spohr, G. M., de Quadros, C. S., Mai, S., Menoncin, S., Ternus, R. Z., \& Dalcanton, F. (2015). Estudo da concentração de fécula de mandioca na utilização em filmes biodegradáveis para o recobrimento de tomates. Blucher Chemical Engineering Proceedings, 1 (2), $3254-3261$.

Pereira, G. D. S., Machado, F. L. D. C., Costa \& J. M. C. D. (2014). Aplicação de recobrimento prolonga a qualidade pós-colheita de laranja 'Valência Delta' durante armazenamento ambiente. Revista Ciência Agronômica, 45, 520-527.

Ribeiro, T. P., Lima, M. A. C. D., Trindade, D. C. G. D., Santos, A. C. N. D. \& Amariz, A. (2009). Uso de revestimentos à base de dextrina na conservação pós-colheita de manga ‘Tommy Atkins'. Revista Brasileira de Fruticultura, 31, 343-351. 
Research, Society and Development, v. 11, n. 2, e2211225292, 2022

(CC BY 4.0) | ISSN 2525-3409 | DOI: http://dx.doi.org/10.33448/rsd-v11i2.25292

Scanavaca Júnior, L., Fonseca, N. \& Pereira, M. E. C. (2007). Uso de fécula de mandioca na pó-colheita de manga 'Surpresa'. Revista Brasileira de Fruticultura, 29, 67-71.

Silva, D. F. P., Siqueira, D. L., Matias, R. G. P., Oliveira, S. P., Lins, L. C. R. D. \& Salomão, L. C. C. (2012). Desempenho de filmes comestíveis em comparação ao filme de policloreto de vinila na qualidade pós-colheita de mexericas 'Poncã'. Ciência Rural, 42, 1770-1773.

Tavares, S. (2003). Maturação e conservação do Tangor 'Murcote' (Citrus reticulata Blanco x C. sinensis Osbeck) e da lima ácida 'Tahiti' (Citrus latifolia Tanaka) sob efeito de biorreguladores. Tese de Doutoramento. Piracicaba, Universidade de São Paulo, 115p.

Vale, A. A. S., Santos, C. D. D., Abreu, C. M. P. D., Corrêa, A. D. \& Santos, J. A. (2006). Alterações químicas, físicas e físico-químicas da tangerina 'Ponkan' (Citrus reticulata Blanco) durante o armazenamento refrigerado. Ciência e Agrotecnologia, 30, 778-786.

Vasconcelos, L. H. C., da SIlva, F. A., da Costa, I. C. \& Vasconcelos, R. F. (2020). Avaliação pós-colheita de tangerinas 'Dekopon' submetidas a radiação ultravioleta C, atmosfera modificada passiva e beneficiamento. Research, Society and Development, 9 (6). 